\title{
Pulmonary Haptoglobin and CD163 Are Functional Immunoregulatory Elements in the Human Lung
}

\author{
M. Abdullah ${ }^{a}$ \\ D. Kähler ${ }^{a}$ \\ C. Vock ${ }^{b}$ \\ N. Reiling ${ }^{c}$ \\ C. Kugler ${ }^{\mathrm{e}}$ \\ D. Drömann ${ }^{f}$
}
J. Rupp ${ }^{\text {G H.P. Hauber }}{ }^{d}$
H. Fehrenbach ${ }^{b}$
P. Zabel ${ }^{d, f}$
E. Vollmer ${ }^{a}$
K. Dalhoff ${ }^{f}$
T. Goldmann ${ }^{\text {a }}$

${ }^{a}$ Clinical and Experimental Pathology, ${ }^{b}$ Experimental Pneumology, Inflammation and Regeneration, and Departments of ${ }^{\mathrm{C}}$ Molecular Infection Biology and ${ }^{\mathrm{d}}$ Clinical Medicine, Research Center Borstel, Borstel, eDepartment of Thoracic Surgery, Hospital Grosshansdorf, Grosshansdorf, ${ }^{f}$ Medical University Hospital III, University Lübeck, and ${ }^{9}$ Medical Clinic III, Campus Lübeck/Institute of Medical Microbiology and Hygiene, University of Schleswig-Holstein, Lübeck, Germany

\section{Key Words}

Acute-phase protein · CD163 • Chronic disease · Lung • Haptoglobin • Infection

\section{Abstract \\ Background: The acute-phase protein haptoglobin $(\mathrm{Hp})$ and its receptor CD163 serve as immunomodulators and possess anti-inflammatory besides antioxidant functions. Objectives: To further understand the role of the recently described pulmonary $\mathrm{Hp}(\mathrm{pHp})$ and its receptor CD163 in case of inflammation and infection, $\mathrm{pHp}$ and CD163 were in- vestigated on $m R N A$ and protein level to gain insight into the cellular events taking place upon stimulation with the in- flammatory mediators LPS, Pam3, cytokine IL- 6 and dexa- methasone, and upon infection with respiratory pathogens (Haemophilus influenzae, Streptococcus pneumoniae and Chlamydia pneumoniae) by use of a human ex vivo tissue cul- ture model and cell cultures of A549 and alveolar epithelial cells type II. In addition, pHp and CD163 expression in COPD and sarcoidosis was assessed. Methods: We conducted ex- periments using 942 ex vivo cultured lung samples applying immunohistochemistry, immunocytochemistry, in situ hy-}

\section{KARGER}

Fax +4161306 1234

E-Mail karger@karger.ch

www.karger.com
C) 2011 S. Karger AG, Basel

0025-7931/12/0831-0061\$38.00/0

Accessible online at:

www.karger.com/res bridization, immunofluorescence, real-time PCR, RT-PCR, slot and Western immunoblot analyses with tissue lysates and culture supernatants as well as ELISA and cytometric bead array analyses. Results: This study describes for the first time the expression, regulation and secretion of $\mathrm{pHp}$ and its receptor CD163 in the human lung. The release of soluble mediators from A549 cell line and human monocytederived macrophages was observed indicating that $\mathrm{Hp}$ differentially activates the release of soluble mediators and major chemoattractants. Conclusions: The findings indicate a native function of $\mathrm{pHp}$ and $\mathrm{CD} 163$ as functional pulmonary defense elements due to local expression, regulation and secretion during lung infection and as part of the inflammatory immune response of the respiratory system.

Copyright $\odot 2011$ S. Karger AG, Basel

\section{Introduction}

During the acute phase of inflammatory processes, elevated expression of acute-phase proteins (APP) is characteristic. One of those molecules is hepatic haptoglobin (Hp) occurring in different phenotypes. Its main role is 
capturing hemoglobin to prevent both iron loss and kidney damage during hemolysis [1]. Little is known about the role of APP during local inflammation. In general, they are capable to suppress nonspecific inflammation via suppressed release of pro-inflammatory cytokines in response to inflammatory stimuli; on the other hand, inflammatory cells express receptors for $\mathrm{Hp}[2,3]$. In human hepatic cells, Hp expression requires glucocorticoids and IL-6 [4], whereas in hepatic rat cells Hp expression requires glucocorticoids as well as both IL- 6 and IL-1 [5]. During inflammation, Hp expression and regulation vary in different tissues and species $[6,7]$. Recently, we described for the first time a pulmonary $\mathrm{Hp}$ molecule ( $\mathrm{pHp}$ ) which is synthesized locally in the human lung [8].

It has been shown that CD163, which was identified as an $\mathrm{Hp}$-hemoglobin ( $\mathrm{Hp}-\mathrm{Hb}$ ) scavenging receptor, is synthesized as a $130-\mathrm{kDa}$ glycoprotein belonging to the scavenger receptor cysteine-rich superfamily [1]. It is expressed exclusively in cells of the monocyte lineage; high levels of CD163 were detected in a number of different mature tissue macrophages such as alveolar macrophages (AM) [1]. The regulation of CD163 expression by pro- and anti-inflammatory mediators and the secretion of antiinflammatory cytokines in response to ligand binding to CD163 strongly suggest an immunomodulatory function of CD163. By contrast, TNF- $\alpha$, IFN- $\gamma$ and LPS suppress CD163 [9]. Interestingly, IL-6 induces both Hp and CD163 expression [9]. Up to date, the role of Hp synthesis in extrahepatic tissues is poorly understood. There may be more functions of $\mathrm{Hp}$ and CD163 in the lung than binding to hemoglobin during hemolysis or signaling related to the inflammatory response.

To address these questions, we investigated expression and regulation of $\mathrm{pHp}$ and CD163 mRNA and protein upon stimulation with Gram-positive and Gramnegative bacteria-simulating, microbial antigens (Pam3 and LPS), inflammatory cytokine IL-6, glucocorticoid (dexamethasone, DEX) and respiratory pathogens (Haemophilus influenzae, Streptococcus pneumoniae and Chlamydia pneumoniae), applying a human ex vivo tissue culture model (short-term stimulation of tissues, STST) in combination with the HEPES-glutamic acid buffermediated organic solvent protection effect (HOPE) fixation technique [10]. The HOPE technique is a novel formalin-free fixation procedure for cells and tissues which results in better preservation of nucleic acids and proteins compared to conventional formalin fixation, while the morphology of HOPE-fixed tissues is comparable to formalin-fixed materials. In addition, the release of soluble mediators and chemoattractant cytokines upon Hp costimulation was investigated. To further illuminate the role of pHp and CD163 in the context of lung inflammation, tissues from patients with chronic obstructive pulmonary diseases (COPD) and sarcoidosis were included. These tissues were not cultivated and diagnoses were confirmed by two pathologists. A functional role for glycoproteins in the regulation of inflammatory processes had already been shown $[11,12]$. The present study holds the hypothesis that synthesis and secretion of high levels of $\mathrm{pHp}$ and CD163 during infection of tissues and cell cultures is an integral part of respiratory immune reactions.

\section{Material and Methods}

\section{Sample Origin and Collection}

Eighty-two human lung tissues were obtained from patients directly after lobectomy/pneumonectomy. Nine hundred fortytwo definitely tumor-free lung tissues of up to $1 \mathrm{~cm}^{3}$, resected distant from the tumor, were collected from those patients and subjected to ex vivo tissue cultivation and stimulation including the respective medium-controls. Further, two pulmonary cell types (alveolar epithelial cells type II, AEC II, primary cells, obtained from the University of Freiburg, Department of Pneumology, and A549 cell line obtained from the Medical Clinic Borstel) were included in this study. For additional characterization of $\mathrm{pHp}$ and CD163 in chronic diseases, we also analyzed 11 COPD and 11 sarcoidosis cases which were not cultivated in STST (see below). Ten HOPE-derived single sections of lung tissue stimulated with inflammatory stimuli were produced additionally for in situ hybridization to verify immunohistochemistry (IHC) results.

This study was approved by the ethical committee of the University of Lübeck (reference No. 03/158) and is in compliance with the Helsinki declaration.

\section{Short-Term Stimulation of Tissues}

We investigated the 942 lung tissue specimens as previously described [13] within the STST model in the following way. For pHp investigations, 14 specimens were stimulated with IL-6 (50 ng/ml; Sigma-Aldrich Chemie $\mathrm{GmbH}), 80$ specimens with LPS (10, 50 and $200 \mathrm{ng} / \mathrm{ml}$; EMC Microcollections $\mathrm{GmbH}), 57$ specimens with Pam3 (200 ng/ml; EMC Microcollections GmbH) and 63 specimens with DEX (10 and $50 \mathrm{ng} / \mathrm{ml}$; Merck) using medium controls (culture medium without stimulus). Ninety-four specimens were pre-incubated for $3 \mathrm{~h}$ in medium to wash the tissue from blood and pre-existent $\mathrm{pHp}$ which was not synthesized due to applied stimulation.

For investigation of CD163 we stimulated 101 specimens with LPS, 67 specimens with DEX, 75 specimens with Pam 3 and 14 specimens with IL- 6 accompanying medium controls. Additionally, ex vivo infection was performed by application of $H$. influenzae, C. pneumoniae and S. pneumoniae into the culture medium (10 specimens each). Culture conditions were set as previously described $\left(37^{\circ} \mathrm{C}\right.$ and $\left.5 \% \mathrm{CO}_{2}\right)$ applying different concentrations 
and incubation periods for each stimulus [14]. Stimulation was performed for $10 \mathrm{~min}, 30 \mathrm{~min}, 2 \mathrm{~h}$ and $24 \mathrm{~h}$. In each STST experiment two specimens were incubated in parallel dedicated either for HOPE or formalin fixation.

Several STST culture supernatants were analyzed using slot immunoblots (see below) to identify secreted pHp and CD163.

\section{Tissue Microarrays and Single Sections}

After stimulation all specimens were formalin-fixed and arranged in tissue microarrays (TMAs) as described previously [10]. Sections from these paraffin blocks were mounted on SuperFrost+ slides (Menzel-Gläser). Twenty-one TMAs were produced containing all differently stimulated tissues.

\section{IHC and Immunocytochemistry}

IHC stainings were performed with formalin-fixed and paraffin-embedded tissue. TMAs or single sections were used as previously described $[8,10]$; the primary mouse anti-human antibodies utilized in this study were a monoclonal anti-Hp antibody (clone HG-36, dilution 1:100; Abcam) and a monoclonal mouse anti-human anti-CD163 antibody (clone 10D6, dilution 1:100; Diagnostic BioSystems) and had been applied for $60 \mathrm{~min}$ at room temperature. Immunocytochemistry was carried out with either HOPE- or formalin-fixed cytospins as previously described [15]. The specificity of the antibody had been shown in a previous report. Immunostaining procedures had also been described previously [8].

\section{Collection of Supernatants}

To investigate secretion of $\mathrm{pHp}$ and $\mathrm{CD} 163$ from ex vivo tissue culture, A549 cell line and AEC II, $2 \mathrm{ml}$ of the culture supernatants were collected and centrifuged at $6,000 \mathrm{rpm}$ for $5 \mathrm{~min}$ before storing at $-80^{\circ} \mathrm{C}$. For pHp investigation $45,32,22$ and 12 supernatants were analyzed following LPS, DEX, IL- 6 and Pam 3 stimulation, respectively. For CD163 analyses 16, 12, 12 and 21 supernatants were used, respectively.

Slot and Western immunoblot analyses were conducted subsequently after application of different concentrations of stimuli $(10,50$ and $200 \mathrm{ng} / \mathrm{ml})$ and incubation times $(10 \mathrm{~min}, 30 \mathrm{~min}, 2 \mathrm{~h}$ and $24 \mathrm{~h}$ ); total protein analyses were performed using Bradford Coomassie Protein Assay (Pierce). Optical density was measured at $595 \mathrm{~nm}$ using a Helios $\beta$-photometer (Unicam).

\section{Slot Immunoblot Analyses of Culture Supernatants}

Immunoblotting of supernatants from ex vivo tissue culture, A549 cell line and AEC II, was performed with PR 60 and PR 648 slot immunoblot filtration manifolds and a vacuum pump (Amersham Biosciences); $300 \mu \mathrm{g}$ per slot were applied, the procedure included four main steps starting from setting up the slot immunoblot, applying samples, removing the blot and immunostaining (analogue to SDS-PAGE Western blot staining). For evaluation of band intensity, a software was used (Band Leader).

\section{Cellular Transcription of pHp within STST Model (in situ} Hybridization)

For confirmation of $\mathrm{pHp}$ transcription in human lung tissues, we analyzed 10 IL-6- stimulated specimens and medium controls for comparison by in situ hybridization (ISH) to detect cellular transcripts of the $\mathrm{pHp}$ mRNA within the tissues. ISH targeting the 341-bp Hp transcript was carried out using the following Hp- specific primers: forward 5'-AGGCATTATGAAGGCAGCAC-3', reverse 3 '-CTTCCAGGCTGAAATCTTGC-5'. Slides were kept in moist chambers overnight at $46^{\circ} \mathrm{C}$. Probes were generated and hybridized like previously described [14].

\section{Conventional RT-PCR}

Total RNA was extracted from 10 human, $H$. influenzae-infected, STST-cultured, HOPE-fixed lung tissues which showed upregulation in IHC stainings (following RNeasy Mini kit; Qiagen). RTPCR was performed conventionally according to Abdullah et al. [8] using Hp-specific primers: forward 5'-AGGCATTATGAAGGCAGCAC-3', reverse 3'-CTTCCAGGCTGAAATCTTGC-5'; for CD163, specific forward primer 5'-AGACCTGGACTATTAAATGC-3', reverse primer 3'-CGTTGTGAGTCATGCCTTAT-5'. In parallel, specific primers for the housekeeping gene glyceraldehde-3-phosphate-dehydrogenase (GAPDH; forward primer 5'AGAACGGGAAGCTTGTCATC-3', reverse primer 3'-TGCTGATGATCTTGAGGCTG-5') were used as controls. For densitometry, Band Leader software was used.

\section{Real-Time RT-PCR}

RNA isolation and cDNA synthesis were done like mentioned above. Amplification was performed using a Light Cycler ${ }^{\circledR}$ carousel-based system (Roche Molecular Biochemicals) with light cycler software 4 LL.2.0 for data analysis. Specific primers against human Hp were used (QuantiTect primer assay; Qiagen). GAPDH was used as housekeeping gene. Primers were as follows: $5^{\prime}$ GGAGTCGGACCCCATATCAT-3' (forward) and 3'-CTGAGAGTCAGGAAGGCACC-5' (reverse). Relative mRNA quantification was done by using standard curves for Hp and GAPDH. The mRNA level in each sample is described as a relative expression value and is compared to the expression level of infected and stimulated lungs out of STST.

\section{Measurement of Chemoattractants and Soluble Mediators} via ELISA and Cytometric Bead Array

To investigate the role of $\mathrm{pHp}$ during the initial local response to the inflammatory stimulus LPS (200 ng/ml), A549 cell's chemokine production and monocyte chemoattractant protein-1 (MCP-1) and CXC (IL-8) were measured. A549 cells were cultured up to $30 \mathrm{~h}$ in the presence or absence of LPS (200 ng/ml) and Hp protein $(150 \mathrm{ng} / \mathrm{ml}$ ) (lyophilized Hp; Abcam). Supernatants were collected at the end of incubation and stored at $-80^{\circ} \mathrm{C}$ until use. Chemokine levels in supernatants were determined using a commercially available ELISA kit for MCP-1 (R\&D Systems GmbH). For IL-8 determination the BioSourceCytoSet kit was used. Optical density measurements were performed with a microplate reader model $450 \mathrm{~nm}$ and values were analyzed with Microplate Manager/PC Data Analysis software (both from Bio-Rad Laboratories). Human monocyte-derived macrophages were generated and used for cytometric bead array (CBA) analysis as previously described [16]. Supernatants from macrophage cultures were analyzed by CBA (BD Biosciences), as recommended by the manufacturer.

\section{SDS-PAGE and Western Blotting}

We performed Western blotting using STST-cultured, HOPEfixed material. Supernatants of cell cultures from the A549 cell line as well as from bronchoalveolar lavage (BAL) stimulated with inflammatory stimuli were additionally blotted. Deparaffiniza- 
Fig. 1. The figure summarizes the effects of LPS, DEX, IL- 6 and Pam3 on the expression $(\mathbf{a}, \mathbf{b})$ and secretion $(\mathbf{c}, \mathbf{d})$ of either pHp or CD163 in ex vivo cultured and stimulated human lung tissues. The $\mathrm{x}$-axes show the numbers of tissues which were stimulated and compared to medium controls. Expression of pHp and CD163 was determined semiquantitatively by IHC, while secretion was analyzed by slot immunoblots using the respective tissue culture supernatants. $\mathbf{a}, \mathbf{b}$ Induction of $\mathrm{pHp}$ and CD163 expression compared to medium controls; given are the percentages of tissues which show a clear induction in IHC stainings. c, d Induction of $\mathrm{pHp}$ and CD163 secretion compared to medium controls, determined by densitometry. The percentages of supernatants showing a clear induction of the secretion compared to medium controls are displayed. $\chi^{2}$ tests were used for statistical analyses $\left.{ }^{*} \mathrm{p}<0.05 ;{ }^{* *} \mathrm{p}<0.01 ;{ }^{* * *} \mathrm{p}<0.001\right)$.

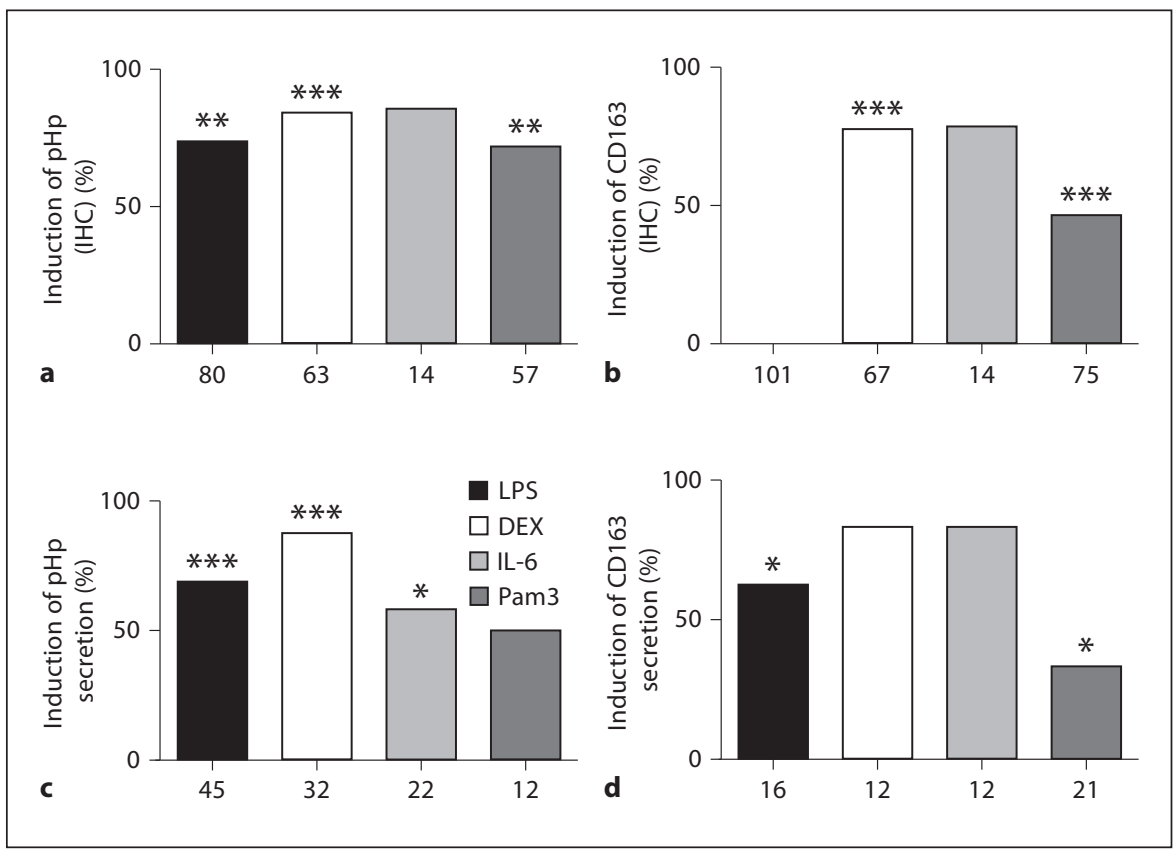

tion, rehydration, extraction and immunostaining were performed as described previously [8]. Protein concentrations were determined in extraction buffer using a spectrophotometer with Bradford Coomassie Protein assay kit (Pierce); $10-15 \mu \mathrm{g}$ of protein were used, mixed with sample buffer $(200 \mathrm{~mm}$ Tris- $\mathrm{HCl} \mathrm{pH}$ 8, $20 \mathrm{mM}$ EDTA, 25\% glycerol, 2\% SDS and $0.02 \%$ bromophenol blue) either containing $1 \%$ DTT as reducing agent or without DTT for nonreducing conditions.

\section{Cell Culture and Cytospin Preparation}

Cytospin preparations were prepared as previously described [15], performing HOPE and formalin fixation in parallel for immunocytochemistry and immunofluorescence stainings (described below). Human A549 cells (American Type Culture Collection) and primary AEC II were maintained in modified high glucose DMEM (4.5 g/l) medium with L-glutamine (PPA) supplemented with $1 \%$ of penicillin/streptomycin solution (Gibco/Invitrogen) and 10\% heat-inactivated FCS (PPA). BAL cells/fluid was further cultured in RPMI 1640 with L-glutamine (PPA) applying inflammatory agents, inflammatory cytokines and DEX. After stimulation, cells were washed 3 times in PBS and harvested after 30 h. Finally, cells were resuspended in PBS reaching a concentration of $2 \times 10^{6} / \mathrm{ml}$. Around 50,000 cells were attached to SuperFrost+ microscope slides (Menzel-Gläser) by centrifugation for $5 \mathrm{~min}$ at $450 \mathrm{rpm}$ using high acceleration in a Cytospin 2 centrifuge (Shandon). Afterwards, cells were dried for $10 \mathrm{~min}$ at $37^{\circ} \mathrm{C}$. After overnight fixation at $4^{\circ} \mathrm{C}$ in HOPE solution, cells were incubated with acetone/glyoxal for $1 \mathrm{~h}$, and dehydrated 6 times for 10 min with acetone (all at $4^{\circ} \mathrm{C}$ ), followed by two incubation periods in isopropanol $\left(10 \mathrm{~min}\right.$ at $60^{\circ} \mathrm{C}, 2 \mathrm{~min}$ at $\left.60^{\circ} \mathrm{C}\right)$ and subsequent air-drying. Rehydration was achieved by incubation in $70 \%(\mathrm{v} / \mathrm{v})$ acetone for $10 \mathrm{~min}$ at $4^{\circ} \mathrm{C}$ and DEPC water incubation for $10 \mathrm{~min}$ (also $4^{\circ} \mathrm{C}$ ). Slides were then air-dried again. For comparative studies, dried cytospins were fixed in $4 \%$ formalin for $10 \mathrm{~min}$ at $4^{\circ} \mathrm{C}$.

\section{Immunofluorescence Staining}

Immunofluorescence stainings of cytospin preparations from BAL (formalin- and HOPE-fixed) were carried out using the same primary antibodies as in the previously described IHC procedure [10]. As a secondary antibody, we applied goat anti-mouse fluorescein isothiocyanate (FITC, Alexa Fluor 488; Invitrogen) in a dilution of 1:200 (45 min in the dark) according to the instructions of the manufacturer. Counterstaining was performed using DAPI (Vector Laboratories) for examination with a fluorescence microscope (Nikon Eclipse 80i); slides were stored at $4^{\circ} \mathrm{C}$ in the dark.

\section{Statistics}

We have included standard errors of the means and $\mathrm{p}$ values from $\chi^{2}$ tests (fig. 1, 4, 6, 10).

\section{Results}

pHp Is Expressed and Regulated by Microbial Antigens, IL-6 and Glucocorticoids in the Human Lung

The numbers of investigated cases (n) and the percentages of cases which showed upregulation of $\mathrm{pHp}$ upon stimulation with different stimuli are shown in figure 1 . All stimuli caused upregulation of $\mathrm{pHp}$ in different intensities and were determined for LPS in $67 / 80$ cases $(83.7 \%)$, for DEX in 54/63 (85.7\%), for IL-6 in 12/14 (85.7\%) and for Pam 3 in 41/57 cases (71.9\%). The baseline of pHp expression in a medium control is displayed in figure $2 \mathrm{e}$, as well as examples of various other stimulation experiments. 


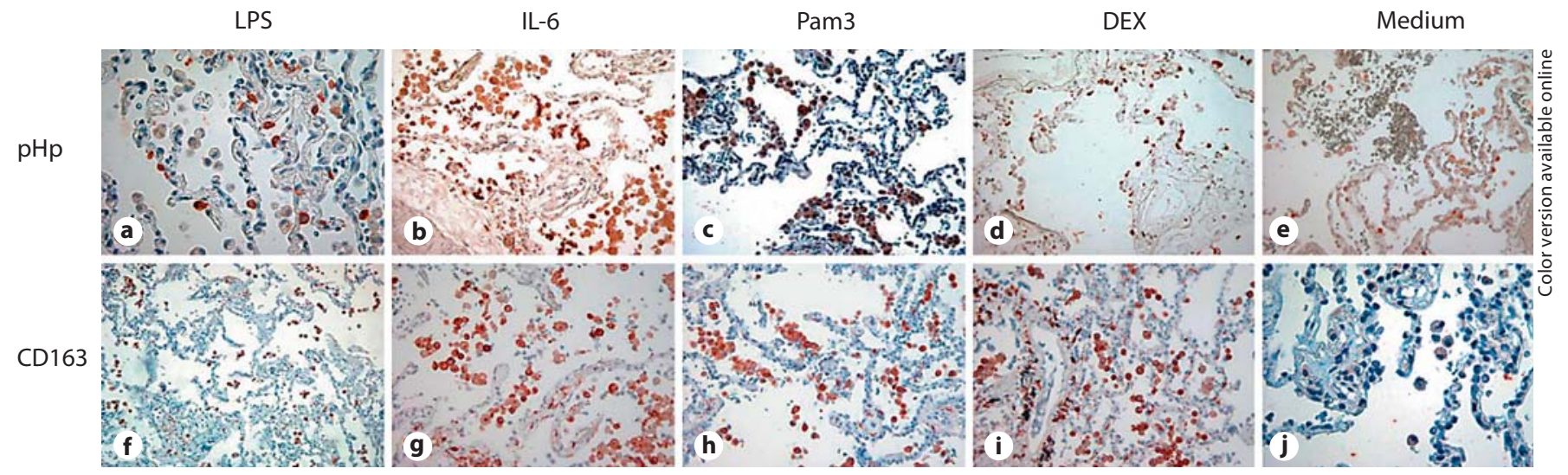

Fig. 2. Regulation of $\mathrm{pHp}(\mathbf{a}-\mathbf{e})$ and CD163 (f-j) in response to different stimuli using the human STST ex vivo tissue culture model. $\mathrm{pHp}$ and CD163 were detected by IHC. Tissues were cultured, stimulated and investigated after $10 \mathrm{~min}, 30 \mathrm{~min}, 2 \mathrm{~h}$ and $24 \mathrm{~h}$. All stimuli caused a time-dependent induction of $\mathrm{pHp}$. $\mathrm{Pam} 3$ and IL- 6 were used in one concentration only, LPS and DEX also displayed a dose dependency. LPS concentration: 10, 50 and $200 \mathrm{ng} /$ $\mathrm{ml}$; Hp staining with $50 \mathrm{ng} / \mathrm{ml}$ LPS after $24 \mathrm{~h}$ (a). IL-6- (50 ng/ml) induced $\mathrm{pHp}$ expression after $24 \mathrm{~h}$ is shown in b. Pam 3 was dosed at $200 \mathrm{ng} / \mathrm{ml}$; Hp expression after $24 \mathrm{~h}$ (c). DEX (tested in 10 and
$50 \mathrm{ng} / \mathrm{ml}$ concentration) showed expression after $24 \mathrm{~h}$; low-dose effect (d). For CD163, the same stimuli and concentrations were used as in pHp stimulation (f-j). f Expression due to $200 \mathrm{ng} / \mathrm{ml}$ LPS after $24 \mathrm{~h}$. g IL-6-induced expression after $24 \mathrm{~h}(50 \mathrm{ng} / \mathrm{ml})$. Expression caused by Pam 3 after $24 \mathrm{~h}$ (h) and effect of $50 \mathrm{ng} / \mathrm{ml}$ of DEX (i) are depicted. Typical examples of medium controls stained for $\mathrm{pHp}(\mathbf{e})$ and CD163 (j) are shown. After stimulation, all tissues were formalin-fixed, paraffin-embedded and sectioned for stainings. All images $200 \times$.
The main sites of protein expression were observed in AEC II and AM. Dose- and time-dependent induction of pHp was observed with DEX and LPS. Pam3 and IL-6 were tested each in one concentration only and induced pHp time dependently. Generally, a granular staining of pHp inside the cytoplasm was observed (fig. 2a-e).

\section{CD163 Is Differently Regulated in Human Lung Cells}

by Microbial Antigens, IL-6 and Glucocorticoids

Figure $1 \mathrm{~b}$ shows the numbers of investigated cases and the related percentages of cases showing an induction of CD163 being differently regulated upon different stimuli in STST. Increased expression of CD163 was caused by DEX, IL-6 and Pam3 in 77.6\% (52/67), 78.5\% (11/14) and $46.6 \%(35 / 75)$ of cases. LPS downregulated receptor expression in $47.5 \%$ of tissues (48/101; fig. 1b). All effects except of Pam3 and IL-6 (only one concentration used) were dose and time dependent. Signals of CD163 were exclusively localized in the cell membranes of AM.

\section{pHp and CD163 Get Actively Secreted from Lung}

Tissues upon Stimulation and Enhance the Secretion

of Soluble Mediators

Figure $1 \mathrm{c}, \mathrm{d}$ shows the total number of investigated cases and in how many cases (in percent) secreted pHp and CD163 could be detected in culture mediums derived from STST. After $10 \mathrm{~min}$ of STST, activation of $\mathrm{pHp}$ protein secretion occurs compared to the medium control (pre-incubated in medium for $3 \mathrm{~h}$; fig. $3 \mathrm{a}$ ). pHp signaling on slot immunoblots after $24 \mathrm{~h}$ of incubation showed intense bands of pHp caused by LPS in 31/45 supernatants (68.8\%), by DEX in $28 / 32$ (87.5\%), by IL-6 in 7/12 (58.3\%) and by Pam 3 in $11 / 22$ supernatants (50\%). Strong signals appeared related to specimens which were not incubated in medium before stimulation (fig. $3 b$ ). An increase in signal intensity correlates with the length of the respective incubation period. Secretion of CD163 was induced by application of LPS in 10/16 (62.5\%), of DEX in 10/12 (83.3\%), of IL-6 in 10/12 (83.3\%) and of Pam3 in 7/21 (33.3\%) cases (fig. 1d). As expected, no secretion was observed in AEC II and A549 culture supernatants as CD163 is associated only to AM.

\section{Hp Induces the Release of Immunomodulatory \\ Molecules in Lung Cells}

Supernatants analyzed from A549 cell line showed that application of pure Hp protein and LPS as well as costimulation with Hp and LPS enhances the release of the soluble chemoattractants IL- 8 and MCP-1 after $30 \mathrm{~h}$ of stimulation. LPS and $\mathrm{Hp}$ alone increase concentrations of 
Fig. 3. Slot immunoblots showing that $\mathrm{pHp}$ gets secreted from human lung tissues upon stimulation with DEX. To wash the tissue and remove pre-existing Hp protein, the specimens were pre-incubated in medium containing $10 \%$ fetal calf serum for $3 \mathrm{~h}$ before DEX (50 $\mathrm{ng} / \mathrm{ml})$ was applied (a). The effect of stimulation was compared by analysis of immediately stimulated tissue without pre-incubation in medium (b). After stimulation with DEX (50 $\mathrm{ng} / \mathrm{ml}), \mathrm{Hp}$ was analyzed by slot immunoblots after $10 \mathrm{~min}, 30 \mathrm{~min}$ (only in preincubation), $2 \mathrm{~h}$ and $24 \mathrm{~h}$. Signal intensities were determined by Band Leader software (densitometry).
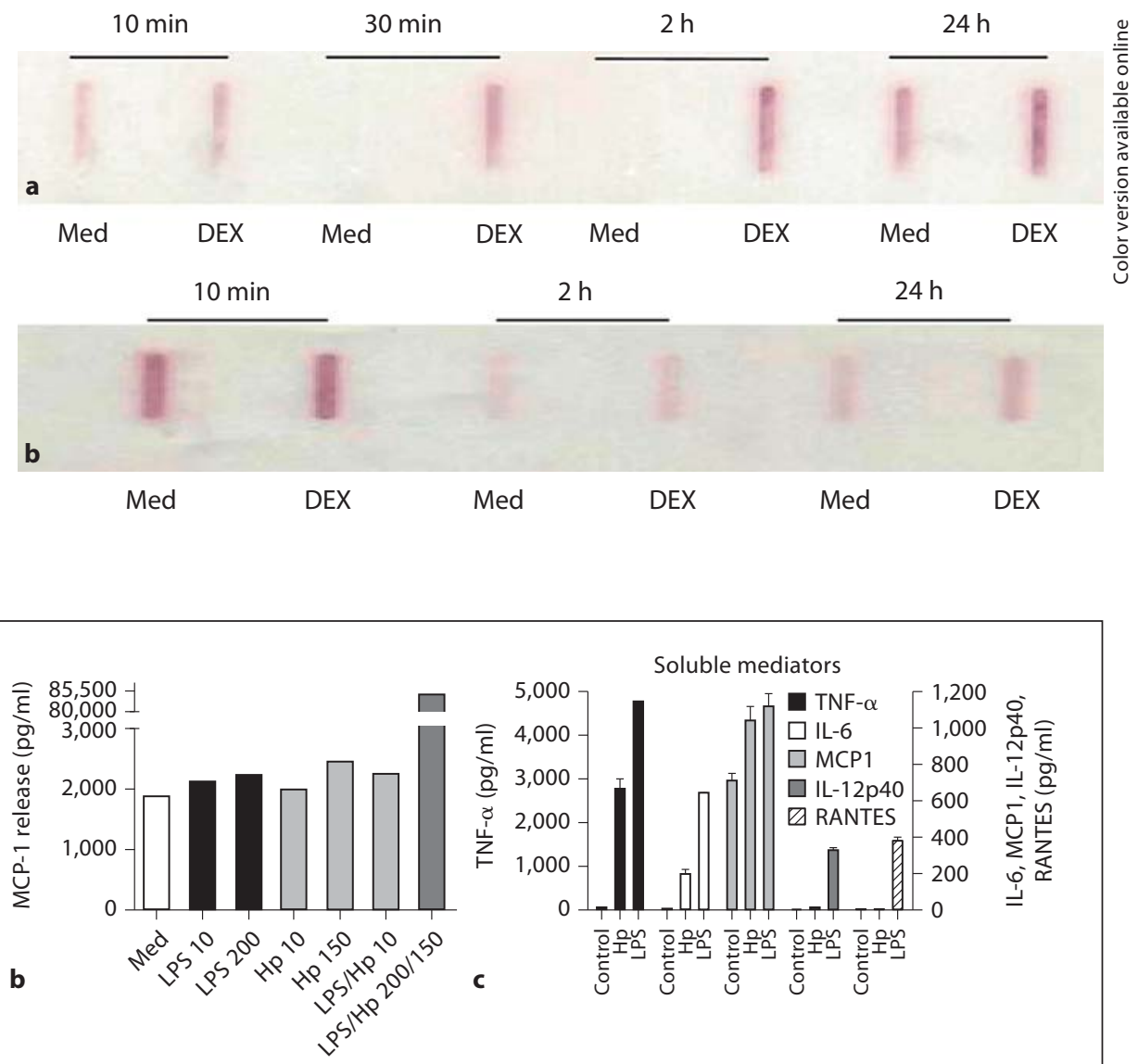

Fig. 4. Cytokines are released into the culture supernatants after stimulation with LPS and Hp protein. a, b Evaluation of the concentrations in A549 cell culture supernatants was measured in medium control and A549 culture supernatants after stimulation with LPS 10 and LPS 200 (10 and $200 \mathrm{ng} / \mathrm{ml}$ ) and pure Hp protein (Hp 10 and $\mathrm{Hp} 150$ for 10 and $150 \mu \mathrm{g} / \mathrm{ml}$ ) as well as LPS and Hp costimulation (both 10 and LPS/Hp $200 \mathrm{ng} / \mathrm{ml}$ and $150 \mu \mathrm{g} / \mathrm{ml}$ ). y-Axes show concentrations of IL-8 (a) and MCP-1 (b) in pg/ml; $\mathrm{x}$-axes show the stimulus with and without co-stimulation by $\mathrm{Hp}$ protein as well as the respective concentrations. The measure-

these chemoattractants dose dependently. Costimulation with LPS and Hp shows a comparably small effect with $10 \mathrm{ng} / \mathrm{ml} \mathrm{LPS}$ and $10 \mu \mathrm{g} / \mathrm{ml} \mathrm{Hp}$, while stimulation with high doses of both $(200 \mathrm{ng} / \mathrm{ml}$ LPS and $150 \mu \mathrm{g} / \mathrm{ml} \mathrm{Hp})$ result in extremely elevated IL- 8 and MCP-1 concentrations (fig. 4a, b).

In human monocyte-derived macrophages, treatment with $10 \mu \mathrm{g} / \mathrm{ml}$ pure Hp protein for $24 \mathrm{~h}$ led to the release of TNF- $\alpha$, IL-6 and MCP-1. In contrast, IL-12p40 and RANTES formation were only detectable in response to LPS but not in response to Hp protein (fig. 4c). In addi- ments were performed using commercial ELISA kits after $30 \mathrm{~h}$ of stimulation and in medium controls. The means of double experiments with two different A459 cell lines are given. c Release of soluble mediators of human monocyte-derived macrophages treated with LPS and pure Hp protein. Macrophages were treated with $\mathrm{Hp}(10 \mu \mathrm{g} / \mathrm{ml})$ or LPS $(10 \mathrm{ng} / \mathrm{ml})$. Supernatants were collected after $24 \mathrm{~h}$ and analyzed by CBA analysis. Data illustrated are representative of two separate experiments with cells from different donors. Means of duplicates \pm SEM are shown. tional in vitro co-stimulation macrophages were treated with $\mathrm{Hp}$ in the presence of LPS. However, the addition of $\mathrm{Hp}$ did not modulate the macrophage response towards these macrophage-activating stimuli (data not shown).

\section{Cellular Transcription of $p H p$}

By application of ISH, $\mathrm{pHp}$ transcripts were found in AM and AEC II of the lung tissues, negative controls did not show any signals (fig. $5 \mathrm{a}-\mathrm{c}$ ). This is in accordance with the cell types found to be expressing $\mathrm{pHp}$ protein by IHC. 


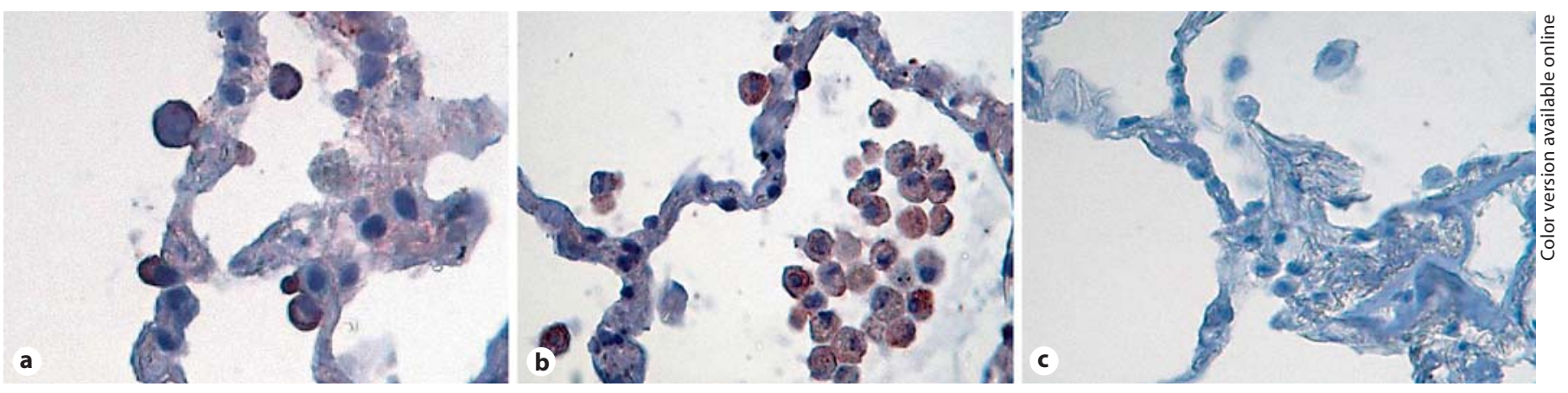

Fig. 5. mRNA transcripts of pHp detected by ISH using a 341-bp digoxigenated, double-stranded DNA-probe on IL-6-stimulated, HOPE-fixed lung tissues. Cellular transcripts were found in AEC II (a) and AM (b) compared to a negative control under omission of the $\mathrm{pHp}$ probe (c).

Transcriptional Regulation of pHp and CD163 in Response to Different Stimuli

In real-time PCR upregulation of $\mathrm{pHp}$ mRNA by DEX, LPS, Pam3 and IL-6 was determined; hereby only DEX affected tissues in a dose- and time-dependent manner (fig. 6a). Concerning LPS, the amount of $\mathrm{pHp}$ mRNA was recorded after stimulation with $200 \mathrm{ng} / \mathrm{ml}$ for $24 \mathrm{~h}$ and showed the highest increase in pHp mRNA. Furthermore, CD163 mRNA expression increases dose dependently according to RT-PCR data in DEX-stimulated lungs (fig. 7a). No variation was observed after Pam3 application; LPS caused downregulation of CD163 which verifies the IHC results (data not shown).

\section{Transcriptional Regulation of $\mathrm{pH}$ and CD163 in}

Response to Infection and Chronic Diseases

RT-PCR showed equal levels of pHp mRNA in all cases. CD163 was significantly downregulated by H. influenzae (fig. 7b). The same is true in real-time PCR, which again shows a downregulation of CD 163 mRNA due to H. influenzae and C. pneumoniae (fig. 6b). A significant upregulation of $\mathrm{pHp}$ was observed in the chronic diseases COPD and sarcoidosis (fig. 6c).

\section{pHp and CD163 Are Induced by Experimental \\ Respiratory Infection}

IHC results revealed elevated expression of $\mathrm{pHp}$ and downregulation of CD163 expression in 11 specimens after 24-hour infection with $H$. influenzae. Expression of $\mathrm{pHp}$ was more frequent in macrophages than AEC II. $S$. pneumoniae caused comparable elevated expression of pHp and CD163 in AEC II and AM (all 11 specimens). Strong upregulation of $\mathrm{pHp}$ and its receptor upon $C$. pneumoniae infection was observed after $24 \mathrm{~h}$, again in AEC II and AM (fig. 8a-d).

\section{Patterns of $p H p$ and CD163 Expression in}

\section{Inflammatory Lung Diseases}

For comparison of $\mathrm{pHp}$ and $\mathrm{CD} 163$ expression in COPD and sarcoidosis, 11 cases were analyzed by IHC. Areas around inflamed airways showed strong $\mathrm{pHp}$ and CD163 expression in AM and AEC II from COPD patients (fig. 9a, b). By contrast, weak expression of $\mathrm{pHp}$ and extremely high expression levels of CD163 from cells forming granuloma lesions of sarcoidosis have been observed (fig. 9c, d).

\section{Western Blot Analysis of Stimulated Tissues \\ Targeting $\mathrm{pH}$ p and CD163}

Western blotting of lysates from STST-cultured tissues and the corresponding supernatants from tissue cultures showed comparable amounts of $\mathrm{pHp}$ protein concerning the different stimuli. Bands showed a molecular weight around $160 \mathrm{kDa}$ (representing $\mathrm{Hp} 2-2$ according to nonreducing conditions and as described in the data sheet; data not shown). In cases of BAL supernatant signaling could be generated only after $35 \mathrm{~h}$ stimulation but not after $24 \mathrm{~h}$. Furthermore, Western blotting targeting CD163 in supernatants of BAL culture and cell lysates revealed upregulation due to DEX and IL- 6 stimulation and downregulation with LPS (data not shown).

\section{pHp Stainings of Cytospin Preparations Prepared} from Primary AEC II

After stimulation with $200 \mathrm{ng} / \mathrm{ml}$ LPS, AEC II showed time-dependent expression of $\mathrm{pHp}$. Equal results were 


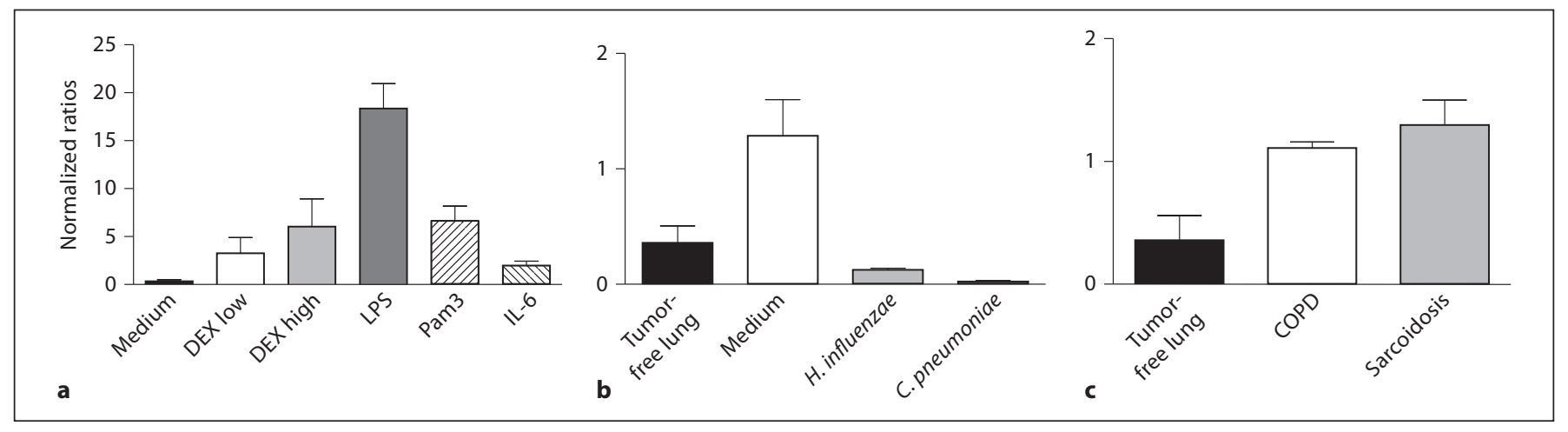

Fig. 6. a, b pHp mRNA levels evaluated by real-time PCR using STST-cultured and -stimulated lung tissues. c pHp mRNA levels in noncultivated, HOPE-fixed tissues ('normal' tumor-free lung, COPD and sarcoidosis). $y$-Axes represent normalized ratios between target calibrator to reference calibrator GAPDH; x-axes indicate the types of tissues which have been analyzed. a STST- stimulated tissues (24 h) with $10 \mathrm{ng} / \mathrm{ml}$ DEX (DEX low), $50 \mathrm{ng} / \mathrm{ml}$ DEX (DEX high), $200 \mathrm{ng} / \mathrm{ml} \mathrm{LPS;} 200 \mathrm{ng} / \mathrm{ml} \mathrm{Pam} 3$ and $50 \mathrm{ng} / \mathrm{ml}$ IL-6. b pHp mRNA levels in non-cultivated tumor-free lung, medium control, $H$. influenzae- and C. pneumoniae-infected lung tissue. c pHp mRNA levels in the tumor-free lung, COPD and sarcoidosis. Means of duplicates \pm SEM are shown.

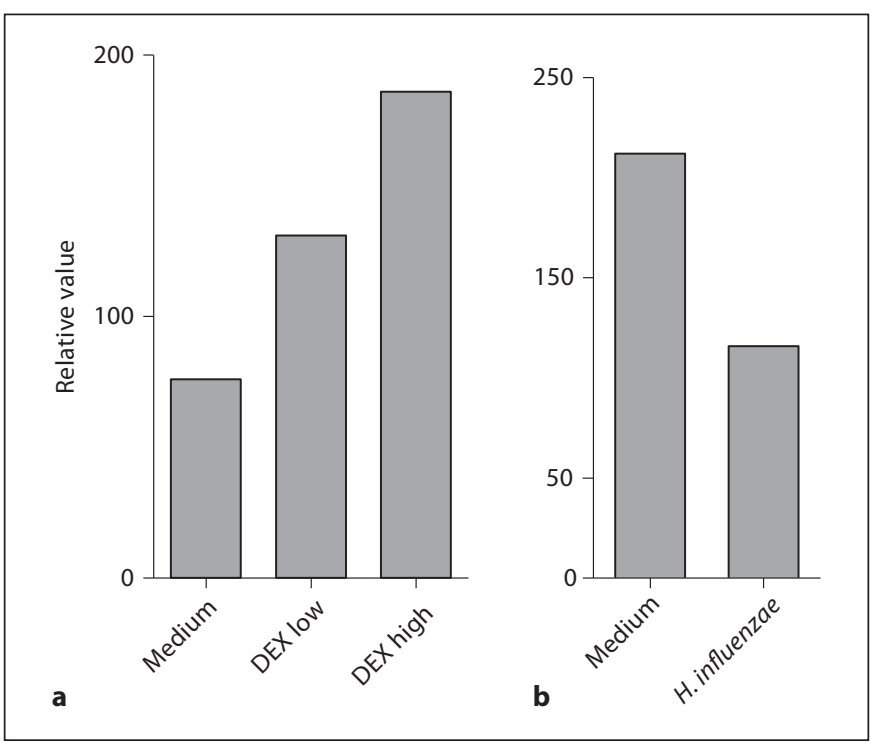

Fig. 7. Detection of CD163 mRNA in stimulated and infected tissues by RT-PCR. Values were determined by densitometry using the software Band Leader. y-Axes show relative values of CD163 amount, $\mathrm{X}$-axes show the stimulation which was performed within the STST model $(24 \mathrm{~h})$ prior to HOPE-fixation. a Medium; DEX low: $10 \mathrm{ng} / \mathrm{ml}$; DEX high: $50 \mathrm{ng} / \mathrm{ml}$. b Medium and H. influenzae.

found after stimulation with DEX and Pam3 (not shown). Figure 10 shows the medium control (fig. 10a) as well as pHp expression after 24 and $35 \mathrm{~h}$ (fig. 10b, c). Figure 10d shows a statistic evaluation of the time-dependent induction of $\mathrm{pHp}$ by LPS.
Immunofluorescence Microscopy Reveals Secretion of pHp via Vesicles due to Stimulation with LPS, DEX and IL-6 in BAL and AEC II Cytospins

Immunofluorescence microscopy of BAL cytospins showed secretion of $\mathrm{pHp}$, the targets were located in vesicles (fig. 11). Further, the immunocytochemistry-positive cytospins prepared from primary AEC II showed pHp induction by DEX, LPS and IL-6 (data not shown, Pam3 was not tested). Expression and secretion in vesicles were observed and clearer in HOPE material compared to formalin; signals were more present after $35 \mathrm{~h}$ compared to $24 \mathrm{~h}$ of stimulation.

\section{Discussion}

The environment of the lung generally needs strong anti-inflammatory properties due to exposure to the atmosphere and a large number of potentially incoming chemical agents, microorganisms, organic and inorganic material or other antigens. Therefore, there have to be effective intra- and extracellular antioxidant defense and other protection mechanisms [17]. If the fast synthesis of gene products related to those purposes is not ensured, lung tissues underlie permanent stress and inflammation. In cases of acute pathogen contact the expression of acute-phase proteins is immediate and local expression becomes inevitable. Recently, pHp has been identified as a part of the lung's local, acute protection system being synthesized locally in lung adenocarcinomas and in the 


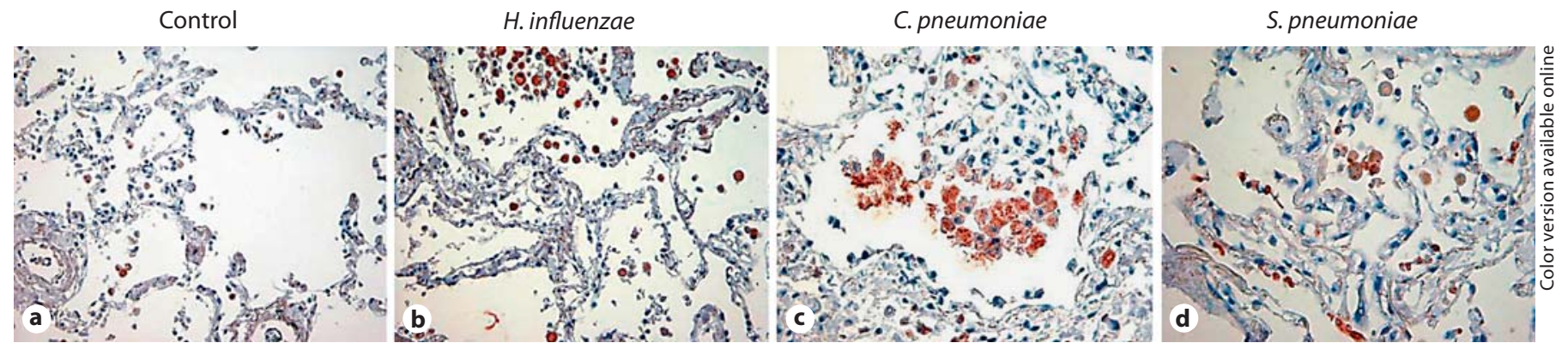

Fig. 8. pHp synthesis is induced by microbial infection with H. influenzae, C. pneumoniae and S. pneumoniae within ex vivo tissue culture after $24 \mathrm{~h}$ of incubation. Here, HOPE technique was applied: medium control without infection (a), infection with $H$. influenzae (b), C. pneumoniae (c) and S. pneumoniae (d). Staining was observed in AM and AEC II. All images $200 \times$.

Fig. 9. Detection of $\mathrm{pHp}$ and $\mathrm{CD} 163$ in chronic lung diseases using IHC. a, b COPD. c, d Sarcoidosis. Concerning COPD, $\mathrm{pH}$ expression was shown within obstructive bronchitis (upper arrow in a) and AM surrounding the obstructive bronchitis (lower arrow in a) while highly elevated CD163 expression from AM (upper arrow in b) was observed. Lower arrow (b) points on the negative obstructive bronchitis for CD163. In case of sarcoidosis, $\mathrm{pHp}$ is expressed weakly in granuloma cells as pointed by lower arrow $(\mathbf{c})$ and most of $\mathrm{AM}$ and AEC II cells surrounding the granulomatous lesions express $\mathrm{pHp}$ (upper arrows in c). CD163 is highly over expressed from cells which form the granulomatous lesions (d). a, b, d $200 \times$. c $400 \times$.

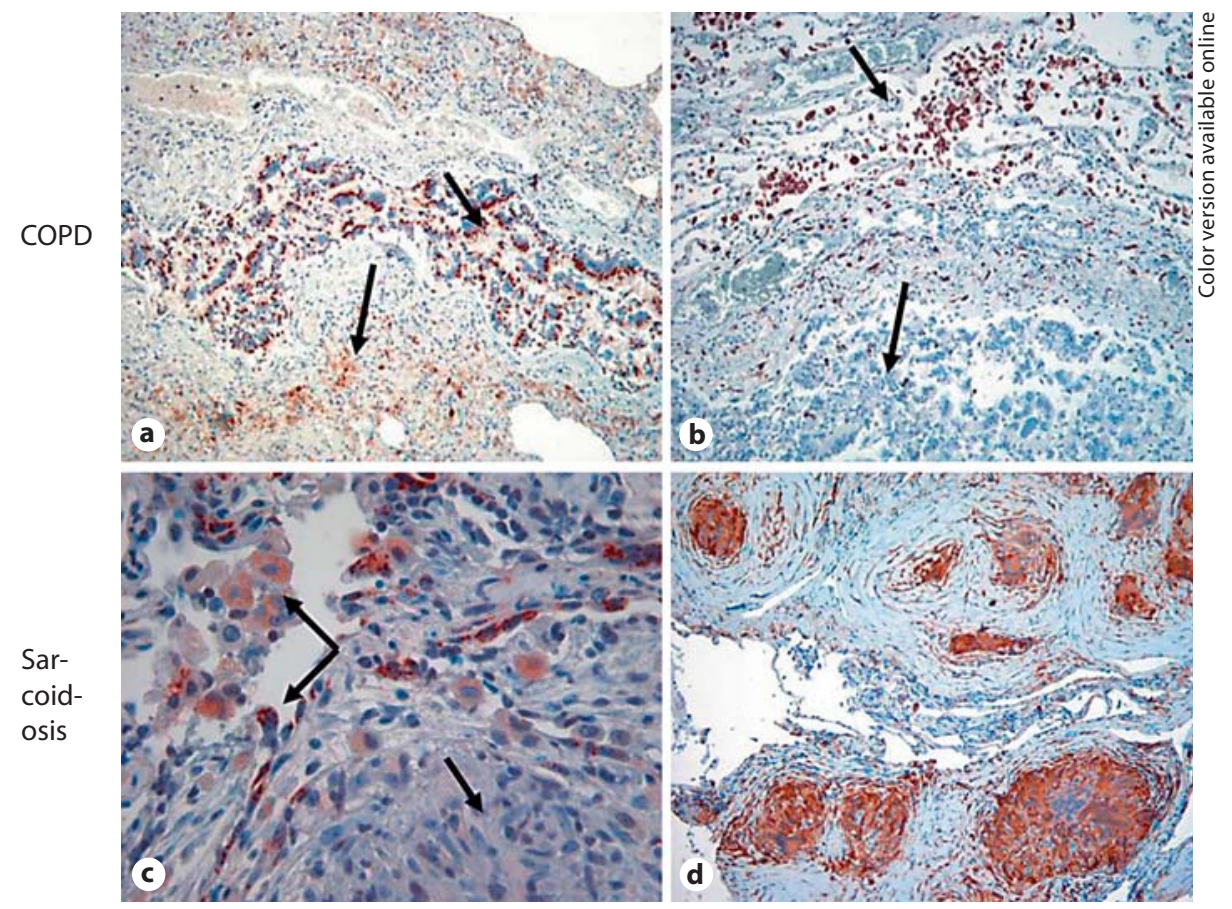

tissue surrounding squamous cell carcinomas. $\mathrm{pHp}$ expression could further be shown in AM and AEC II of tumor-free lung tissue [8].

The enhanced production of APPs in response to inflammatory stimuli suggests that these proteins might augment the acute inflammatory response.

\section{Regulation of $\mathrm{pH} \mathrm{p}$}

In our study, induction of $\mathrm{pHp}$ was shown with inflammatory agents LPS, IL-6 Pam3 and DEX on protein and mRNA level. The results have been verified by data from stimulation-experiments with A549 cells and primary AEC II (IHC).

The induction is caused by many factors and the regulation of $\mathrm{Hp}$ is varying among species and cell types. IL-6, IL-1, TNF- $\alpha$ and glucocorticoids are capable of upregulating the synthesis of $\mathrm{Hp}$ in rat and bovine hepatic cell lines [18].

The activity of DEX on pHp and CD163 is of pharmacologic relevance since DEX is a frequently applied drug in pneumology. In humans, $\mathrm{Hp}$ is mainly regulated by IL- 6 and DEX which is in agreement with results of this study. 


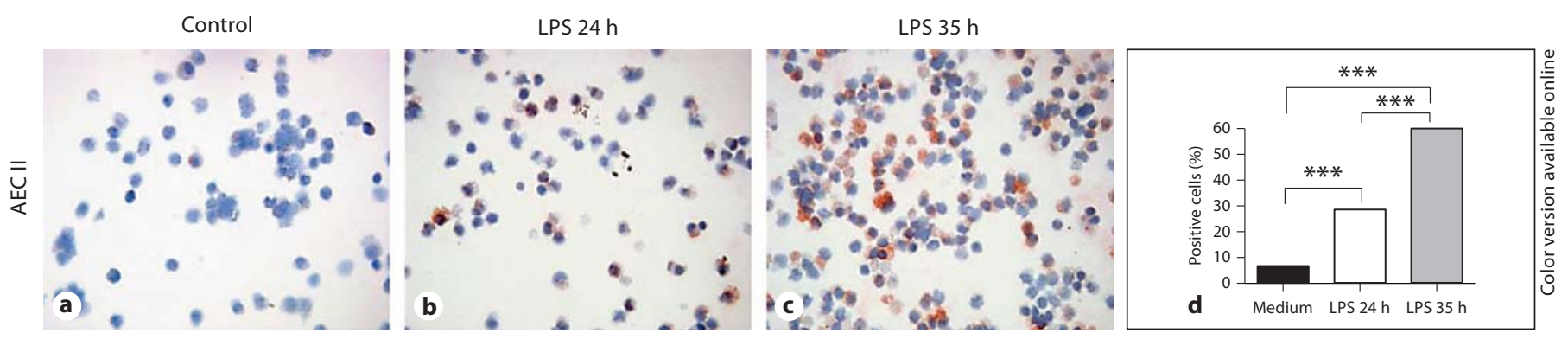

Fig. 10. pHp stainings of cytospin preparations prepared from primary AEC II stimulated with $200 \mathrm{ng} / \mathrm{ml} \mathrm{LPS}$ showing time-dependent expression (same results were found after stimulation with DEX and Pam3). a Medium control. b pHp after 24 h. c pHp after 35 h. d Statistic significance was calculated by $\chi^{2}$ test and Fisher's exact test. Both revealed significant differences between the three groups $\left({ }^{* *} \mathrm{p}<0.001\right)$. All images $\times 200$.
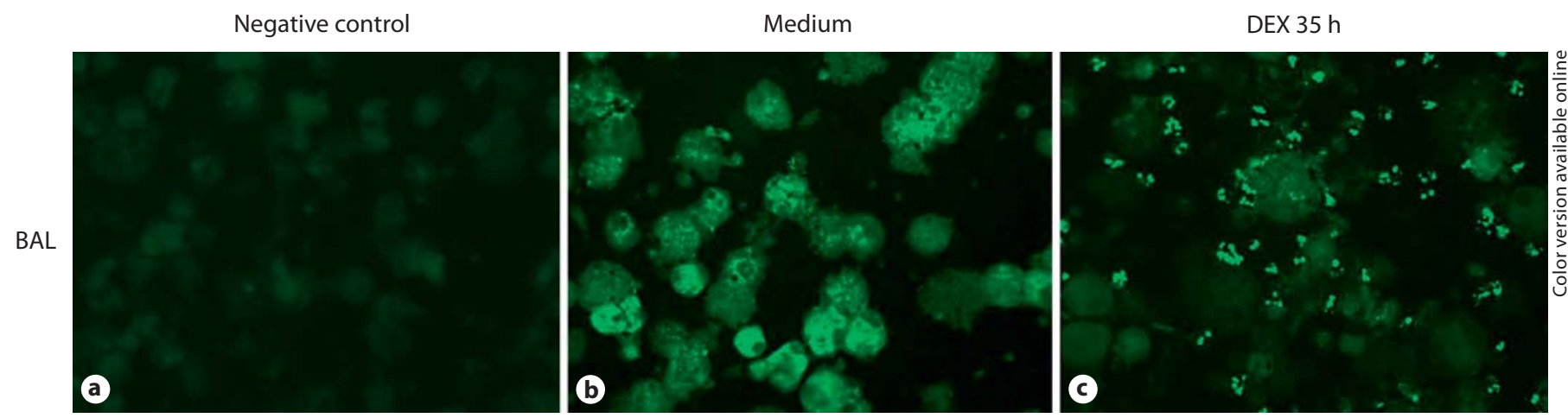

Fig. 11. Immunofluorescence staining of BAL. pHp gets secreted in vesicles near to the macrophages after stimulation with IL-6, LPS and DEX. The images show a negative control under omission of the primary anti-Hp antibody (a), a medium control without stimulation (b) and DEX-treated BAL cytospins secreting $\mathrm{pHp}$ after $35 \mathrm{~h}$ of incubation (c). Immunofluorescence staining was performed on HOPE-fixed BAL using primary monoclonal anti-human Hp antibody; secondary antibody was labeled with FITC. Nuclei were stained with DAPI. All images $\times 400$.

At the first view it seems curious that $\mathrm{pHp}$ is upregulated and released upon apparently 'controversial' stimuli, which moreover takes place in the same time frames. We conclude that $\mathrm{pHp}$ release is not only involved in anti-inflammatory effects of glucocorticoids, but also helps to limit the inflammatory reactions upon bacterial infection. Therefore, we speculate an additional role of $\mathrm{pHp}$ in 'keeping chronic infectious processes chronic' which is supported by the data obtained from COPD and sarcoidosis.

IL-6 is a well-known inflammation-related cytokine, trigger of the acute-phase response and mediator for $\mathrm{Hp}$ gene regulation in all species studied so far $[19,20]$. Hp increases approximately 3 - to 6 -fold in the plasma of mice receiving LPS injections; the regulation of $\mathrm{Hp}$ response to inflammation has been carefully studied in vitro using hepatoma cells which depend on an interaction between specific DNA sequences and a nuclear binding protein (C/EBP- $\beta$ ) for IL- 6 serving as transcription factor for Hp (a sequence in the $5^{\prime}$-regulatory region of the human $\mathrm{Hp}$ gene) [5].

We have shown that exogenous $\mathrm{pHp}$ is stored in monocytes and macrophages within the cytoplasm (granular staining) and other groups reported that it is further secreted by these cells during phagocytosis of Candida albicans [21]. This indicates that $\mathrm{pHp}$ protein levels enhance locally at the sites of inflammation or due to induction by stimuli to modulate granulocyte activity as we have shown in supernatants of ex vivo tissue cultures. Furthermore, specific binding of Hp to neutrophils leads to an inhibition of respiratory burst activity [3]. 
In addition, it has been reported that Hp suppresses macrophage function such as LPS-induced production of TNF- $\alpha$, proliferation and cytokine production by T cells, and proliferation of $\mathrm{B}$ cells. This study shows that there are more differential functions of $\mathrm{pHp}$ to activate monocytes and macrophages and in cell lines as shown in stimulated human derived macrophages. This is in agreement with Arredouani et al. [22]. Hp has further been shown to bind to mono- and polynuclear cells through CD11/ CD18, CD22 and other yet unidentified surface antigens. Therefore, due to the wide distribution of receptors, $\mathrm{Hp}$ modulates functions of these cells and causes endocytosis of $\mathrm{Hp}$ with exerting its immunomodulatory and anti-inflammatory effects through receptor-mediated signaling $[2,22]$.

\section{Regulation of CD163}

This study also describes an induction of CD163 by DEX and IL-6; on the other side, CD163 gets downregulated in AM due to LPS stimulation. This is in agreement with other studies dealing with CD163 in cell lines [9]. The upregulation of CD163 observed in this study is also in agreement with Ritter et al. [23] who showed the presence of multiple glucocorticoid response elements in the promoter region of the human CD163 gene and by describing several consensus binding sites in the sequence. This might be important for the strong expression of CD163 mRNA during IL-10 and IL- 6 treatment $[11,23]$. In contrast to anti-inflammatory stimuli, pro-inflammatory substances decrease CD163 mRNA levels. LPS represents a potent inflammatory stimulus and has been shown to mediate some of its action by members of the Rel-family transcription factors, although there are no consensus sequences for Rel located in the proximal promoter sequence of CD163 [24].

Recently, CD163 was identified as an $\mathrm{Hp}-\mathrm{Hb}$ scavenger receptor [1]. Therefore, increased synthesis of $\mathrm{pHp}$ and CD163 by AM as reaction on inflammatory agents and DEX could contribute to the removal of $\mathrm{Hb}$ and thus protect the lower respiratory tract against $\mathrm{Hb}$-related oxidative damage and inflammation.

Interestingly, CD163 mRNA and protein level show downregulation upon LPS stimulation but high protein levels in supernatants. This effect can be explained by LPS-caused shedding of CD163 from the surface of macrophages (known as soluble form of hemoglobin scavenger receptor sHbSR) [25, 26]. Upregulation caused by DEX and IL-6 highly increased the levels of CD163; this was observed in patients with myelo-monocytic leukemia and infection [25]. An elevated expression of $\mathrm{pHp}$ and CD163 from a local source, and, additionally, systemic $\mathrm{Hp}$ at the site of inflammation or due to induction via inflammatory agents seems to be a scavenging pathway for fast elimination of pro-inflammatory oxidative agents and for the modulation of granulocyte activity.

\section{Fast Secretion of pHp as a Regulatory Strategy}

Transcription data of pHp mRNA and IHC data differ slightly for low-dose LPS (10 and $50 \mathrm{ng} / \mathrm{ml}$ ) stimulation because time and dose dependence could only be observed in IHC. By contrast to LPS, DEX possesses specific responsive elements in the promoter region of the human Hp and CD163 genes [11, 19]. Elevated transcription of $\mathrm{pHp}$ was recorded during high-dosed LPS stimulation $(200 \mathrm{ng} / \mathrm{ml})$ after $24 \mathrm{~h}$, which is in agreement with the results of IHC and slot immunoblot analyses showing fast secretion in a dose- and time-dependent manner. But this might be influenced by secretion of pre-existing $\mathrm{pHp}$ [21], so tissues were washed from pre-existing $\mathrm{pHp}$ by pre-incubation in medium to prove $\mathrm{pHp}$ synthesis definitely due to stimulation. We found that after $10 \mathrm{~min}$ of stimulation secretion starts and increases up to $24 \mathrm{~h}$.

\section{Secreted $p H p$ Induces Immunomodulatory Molecules}

The significant role of $\mathrm{pHp}$ for the secretion of chemoattractants and soluble mediators from the A549 cell line and human monocyte-derived macrophages indicates an important involvement in chemoattractant effects of human lungs and differential activation of the release of pro-inflammatory mediators by human macrophages. This is in agreement with studies which observed that pro-inflammatory cytokines such as TNF- $\alpha$ induce AEC II to produce chemoattractants that could provoke the transendothelial migration of CD3+lymphocytes and CD14+ monocytes [27]. Recent studies also suggest that the alveolar epithelium plays a role in modulating immune responses; the immor talized lung epithelial cell line A549 can produce MCP-1, RANTES and IL-8 following stimulation with TNF- $\alpha$ and other pro-inflammatory cytokines $[28,29]$. Rat type II pneumocytes produce MCP-1 in response to IL-1 [29] and can be stimulated to produce neutrophil chemoattractants [30]. Our results prove for the first time that $\mathrm{pHp}$ in human AEC II contributes to the development of local inflammatory responses by production of the major chemoattractant cytokines MCP-1 and IL- 8 which are responsible for attracting monocytes, lymphocytes and polymorphonuclear cells and soluble mediators. The potentiation of the effects obtained with high doses of Hp+LPS seems to be a possible way for immediate reaction upon acute and massive inflammation. 


\section{pHp and CD163 in COPD and Sarcoidosis}

This study additionally suggests a role of $\mathrm{pHp}$ and CD163 in chronic diseases (such as COPD and sarcoidosis) where strong expression has been observed. Indications summarized lead to speculations that there are differential histological changes in lesions between two chronic cases as well as due to increased levels of chemokines which are responsible for the recruitment of monocytes and for forming chronic inflammation. This is characterized by continuous recruitment and activation of immune cells such as monocytes in response to a persistent stimulus and by keeping the disease in chronic condition because both $\mathrm{Hp}$ and $\mathrm{CD} 163$ are known to serve as immunomodulators during inflammation with elevated levels in the healing phase of acute inflammatory reactions, in chronic inflammatory diseases and during wound healing processes $[11,12]$.

\section{pHp and CD163 in Pulmonary Infections}

Besides antibacterial effects through depleting hemoglobin, which is a main source for bacteria requirements as found in recent studies, deletion of a set of three Hp$\mathrm{Hb}$ binding protein genes from a nontypeable strain (86$028 \mathrm{NP}$ ) of $H$. influenzae attenuated virulence in the chinchilla otitis media model of noninvasive disease [31]. CD163 has been shown to bind both Gram-positive and Gram-negative bacteria and is activated through TLRs. Furthermore, regulation of CD163 during an innate immune response implies an important role for this molecule in host defense against infection [26, 32]. We found $\mathrm{pHp}$ and CD163 downregulated in lung tissues upon $H$. influenzae infection showing decreased mRNA synthesis; the observed high-protein levels might be explained by accumulation of $\mathrm{pHp}$ protein in cellular vesicles. We provide evidence that local $\mathrm{pHp}$ synthesis and secretion are increased after inflammatory induction in STST and alveolar cell lines. The secretion of $\mathrm{pHp}$ by cells is a comparably fast mechanism since $\mathrm{pHp}$ was measured in the supernatants already after 10 min of stimulation, which suggests a high degree of independence from other pathways, for example that of TLRs. Due to the comparably large amounts of quickly secreted $\mathrm{pHp}$ it should be considered that a substantial amount of immunoregulatory events in the human lung are taking place in a $\mathrm{pHp}$-enriched environment.

Further studies will focus on the interaction of CD163 with bacterial ligands, intracellular signaling pathways and actual roles of CD163 and pHp during bacterial infection in vivo and by use of human materials and mouse models (knockout experiments). Furthermore, experiments with knockdown or inhibition of $\mathrm{pHp}$ and CD163 in cell systems are underway to further enlighten the role of this complex in the human lung.

Finally, the results of this study strongly suggest a role of $\mathrm{pHp}$ in antioxidant, inflammation-influencing and immunomodulatory control mechanisms of the human lung. The complete integration of these new results into the complex immunoregulatory network of the lung can contribute to the development of novel treatment regimens facing acute or chronic infections. Finally, these findings confirm a function of $\mathrm{pHp}$ and its receptor as native local pulmonary defense elements with influence beyond the yet known immunomodulatory and antioxidant properties directly within the respiratory system.

\section{Acknowledgements}

The authors thank Jasmin Tiebach, Stefanie Fox, Maria Lammers and Jessica Hofmeister for excellent technical support.

\section{Financial Disclosure and Conflicts of Interest}

The authors declare to have no competing financial interests.

\section{References}

1 Kristiansen M, Graversen JH, Jacobsen C, Sonne O, Hoffman HJ, Law SK, Moestrup SK: Identification of the haemoglobin scavenger receptor. Nature 2001;409:198-201.

$\checkmark 2$ El Ghmati SM, Van Hoeyveld EM, Van Strijp JG, Ceuppens JL, Stevens EA: Identification of haptoglobin as an alternative ligand for CD11b/CD18. J Immunol 1996;156:25422552 . $\checkmark 3$ Oh SK, Pavlotsky N, Tauber AI: Specific binding of haptoglobin to human neutrophils and its functional consequences. J Leukoc Biol 1990;47:142-148.

-4 Wang Y, Kinzie E, Berger FG, Lim SK, Baumann $\mathrm{H}$ : Haptoglobin, an inflammation-inducible plasma protein. Redox Rep 2001;6: 379-385.
5 Marinkovic S, Baumann H: Structure, hormonal regulation, and identification of the interleukin-6- and dexamethasone-responsive element of the rat haptoglobin gene. Mol Cell Biol 1990;10:1573-1583.

6 D’Armiento J, Dalal SS, Chada K: Tissue, temporal and inducible expression pattern of haptoglobin in mice. Gene 1997;195:19-27. 
7 Wang H, Gao XH, Wang YK, Li P, He CD, Xie Y, Chen HD: Expression of haptoglobin in human keratinocytes and Langerhans cells. Br J Dermatol 2005;153:894-899.

8 Abdullah M, Schultz H, Kahler D, Branscheid D, Dalhoff K, Zabel P, Vollmer E, Goldmann T: Expression of the acute phase protein haptoglobin in human lung cancer and tumor-free lung tissues. Pathol Res Pract 2009;205:639-647.

-9 Buechler C, Ritter M, Orso E, Langmann T, Klucken J, Schmitz G: Regulation of scavenger receptor CD163 expression in human monocytes and macrophages by pro- and antiinflammatory stimuli. J Leukoc Biol 2000 67:97-103.

10 Olert J, Wiedorn KH, Goldmann T, Kuhl $\mathrm{H}$, Mehraein Y, Scherthan H, Niketeghad F, Vollmer E, Muller AM, Muller-Navia J: HOPE fixation. a novel fixing method and paraffin-embedding technique for human soft tissues. Pathol Res Pract 2001;197:823826.

-11 Schaer DJ, Boretti FS, Schoedon G, Schaffner A: Induction of the CD163-dependent haemoglobin uptake by macrophages as a novel anti-inflammatory action of glucocorticoids. Br J Haematol 2002;119:239-243.

$\checkmark 12$ Zwadlo G, Voegeli R, Osthoff KS, Sorg C: A monoclonal antibody to a novel differentiation antigen on human macrophages associated with the down-regulatory phase of the inflammatory process. Exp Cell Biol 1987; 55:295-304.

13 Lang DS, Droemann D, Schultz H, Branscheid D, Martin C, Ressmeyer AR, Zabel P, Vollmer E, Goldmann T: A novel human ex vivo model for the analysis of molecular events during lung cancer chemotherapy. Resoir Res 2007;8:43.

-14 Droemann D, Rupp J, Goldmann T, Uhlig U, Branscheid D, Vollmer E, Kujath P, Zabel P, Dalhoff K: Disparate innate immune responses to persistent and acute Chlamydia pneumoniae infection in chronic obstructive pulmonary disease. Am J Respir Crit Care Med 2007;175:791-797.
15 Umland O, Ulmer AJ, Vollmer E, Goldmann T: HOPE fixation of cytospin preparations of human cells for in situ hybridization and immunocytochemistry. J Histochem Cytochem 2003;51:977-998.

16 Reiling N, Blumenthal A, Flad HD, Ernst M, Ehlers S: Mycobacteria-induced TNF-alpha and IL-10 formation by human macrophages is differentially regulated at the level of $\mathrm{mi}$ togen-activated protein kinase activity. J Immunol 2001;167:3339-3345.

17 Miyata R, Van Eeden SF: Treatment of acute lung injury: time to think outside the box? Respiration 2011;81:6-8.

18 Kim H, Baumann H: The carboxyl-terminal region of STAT3 controls gene induction by the mouse haptoglobin promoter. J Biol Chem 1997;272:14571-14579.

19 Friedrichs WE, Navarijo-Ashbaugh AL, Bowman BH, Yang F: Expression and inflammatory regulation of haptoglobin gene in adipocytes. Biochem Biophys Res Commun 1995;209:250-256.

20 Tilg H, Dinarello CA, Mier JW: IL-6 and APPs: anti-inflammatory and immunosuppressive mediators. Immunol Today 1997; 18: 428-432.

-21 Wagner L, Gessl A, Parzer SB, Base W, Waldhausl W, Pasternack MS: Haptoglobin phenotyping by newly developed monoclonal antibodies. Demonstration of haptoglobin uptake into peripheral blood neutrophils and monocytes. J Immunol 1996;156:19891996.

22 Arredouani MS, Kasran A, Vanoirbeek JA, Berger FG, Baumann H, Ceuppens JL: Haptoglobin dampens endotoxin-induced inflammatory effects both in vitro and in vivo. Immunology 2005;114:263-271.

23 Ritter M, Buechler C, Langmann T, Schmitz G: Genomic organization and chromosomal localization of the human CD163 (M130) gene. a member of the scavenger receptor cysteine-rich superfamily. Biochem Biophys Res Commun 1999;260:466-474.

24 Ritter M, Buechler C, Langmann T, Orso E, Klucken J, Schmitz G: The scavenger receptor CD163. regulation, promoter structure and genomic organization. Pathobiology $1999 ; 67: 257-261$.
25 Moller HJ, Aerts H, Gronbaek H, Peterslund NA, Hyltoft PP, Hornung N, Rejnmark L, Jabbarpour E, Moestrup SK: Soluble CD163. a marker molecule for monocyte/macrophage activity in disease. Scand J Clin Lab Invest Suppl 2002;237:29-33.

26 Weaver LK, Pioli PA, Wardwell K, Vogel SN, Guyre PM: Up-regulation of human monocyte CD163 upon activation of cell-surface Toll-like receptors. J Leukoc Biol 2007;81: 663-671.

27 Eghtesad M, Jackson HE, Cunningham AC: Primary human alveolar epithelial cells can elicit the transendothelial migration of CD14+ monocytes and CD3+ lymphocytes. Immunology 2001;102:157-164.

28 VanOtteren GM, Strieter RM, Kunkel SL, Paine R III, Greenberger MJ, Danforth JM, Burdick MD, Standiford TJ: Compartmentalized expression of RANTES in a murine model of endotoxemia. J Immunol 1995;154: 1900-1908.

29 Paine R III, Rolfe MW, Standiford TJ, Burdick MD, Rollins BJ, Strieter RM: MCP-1 expression by rat type II alveolar epithelial cells in primary culture. J Immunol 1993; 150:4561-4570.

30 Rosseau S, Selhorst J, Wiechmann K, Leissner K, Maus U, Mayer K, Grimminger F, Seeger W, Lohmeyer J: Monocyte migration through the alveolar epithelial barrier: adhesion molecule mechanisms and impact of chemokines. J Immunol 2000;164:427-435.

- 31 Morton DJ, Smith A, Ren Z, Madore LL, Vanwagoner TM, Seale TW, Whitby PW, Stull TL: Identification of a haem-utilization protein (Hup) in Haemophilus influenzae. Microbiology 2004;150:3923-3933.

- 32 Fabriek BO, van BR, Deng DM, Ligtenberg AJ, Nazmi K, Schornagel K, Vloet RP, Dijkstra CD, van den Berg TK: The macrophage scavenger receptor CD163 functions as an innate immune sensor for bacteria. Blood 2009;113:887-892. 